\title{
The U.K. Nova/Supernova Search Programme
}

\section{Guy M. Hurst Coordinator}

16, Westminster Close, Kempshott Rise, Basingstoke, Hants, RG22 4PP, U.K.

A summary of the results of the UK Nova/Supernova Patrol is presented together with a brief outline of projects undertaken by our members.

The UK/Nova/Supernova Patrol was formed in $1976 \mathrm{June}$ and the project is run on behalf of the British Astronomical Association, The Astronomer magazine and various overseas groups with the aim of searching for novae, supernovae and to monitor various 'recurrent' objects.

The early successes in 1975/1976 were principally the finding of various pre-discovery images of novae and other eruptive objects on routine patrol films, e.g. : V400 Persei, V373 Scuti, HM Sge. This provided valuable information on the light-curves of such objects and helped to determine the date and magnitude of maximum.

The first major success for the patrol came in 1977 January when John Hosty discovered HS Sagittae (= Nova Sge 1977). The equipment was modest 10 x 50 monocular and from a light-polluted area. This emphasized that expensive equipment was not needed to be successful, just dedication from the observer.

D. Rossiter was unlucky in 1978 when he discovered V1668 Cygni, but after an earlier discovery in USA. M. Swan found pre-discovery images of PU Vul on patrol films in 1979, and John Hosty rediscovery CSV 101897 on films in 1980 October.

The most mysterious patrol discovery occurred in 1981 January when D. Branchett found a nova-like object in Scutum. Although not subsequently confirmed, research showed that the star had been noted in earlier records as an 'atlas omission'. Further deep photographs failed to show a new object to a limiting magnitude of 18 .

The star VY Aquarii, then classified as a recurrent nova, featured in patrol news of 1982-83. In 1982, R. McNaught announced he had found the star of the Papadopoulos Atlas and followed this by observing it at maximum in 1983 November, the first time it had been seen visually. This, and subsequent research by patrol members, led to the star being re-classified as a dwarf nova.

Annick Merlin in France recorded PW Vul (= Nova Vulpeculae 1984) on photographs prior to its discovery announcement 1984 August. Then, in 1985 April, H. Mikuz, operating patrol cameras in Yugoslavia, recorded an outburst of RS Ophiuchi. 
The trend towards monitoring 'recurrent objects' continued and success was achieved with the first-ever visual observation of DO Draconis by S. Lubbock in 1985 October.

A further major success for the patrol came in 1986 with the discovery of Nova Centauri 1986 by R. McNaught, one of our patrollers in Australia.

The same observer provided a pre-discovery series of pictures of the Supernova 1987A in the Large Magellanic Cloud. These results, as with the numerous previous photographs of newly discovered objects, provided invaluable information on the object's rise from minimum.

He followed this with another nova discovery in Sagittarius in 1987 May, the third nova discovery for the patrol.

The above notes provide just a summary of the main achievements but are by no means complete. A detailed report on the results of the project for 1976-1987, together with a full list of bibliography references, will appear in a future issue of the Journal of the British Astronomical Association.

If any observers wish to participate in the search for novae and supernovae, or astronomers require details of our results, please write to the author for further information. 